\title{
Some electrophysical properties of polycrystalline silicon obtained in a solar oven
}

\section{Lutfiddin Omanovich Olimov ( $\nabla$ o.lut@mail.ru )}

ANDIJAN MACHINE-BUILDING INSTITUTE https://orcid.org/0000-0002-0135-5861

Iqboljon Anarboyev

Andijan Machine-Building Institute: Andijon mashinasozlik instituti

\section{Research Article}

Keywords: polycrystalline silicon, microstructure, a solar oven, specific resistance, grain boundary regions, semiconductor

Posted Date: August 3rd, 2021

DOI: https://doi.org/10.21203/rs.3.rs-759058/v1

License: (c) (1) This work is licensed under a Creative Commons Attribution 4.0 International License. Read Full License 


\section{Abstract}

The article describes the results of the study of the microstructure and some electrophysical properties of silicon obtained by re-melting in a solar oven. It was found that the granularity of polycrystalline silicon consists of Si atoms with a size of $10-15 \mu \mathrm{m}$, the roughness of its surface. Decrease in specific resistance at $\mathrm{T} \leq 600 \mathrm{~K}$, increase in concentration of ionized input atoms and concentration of charge carriers, the position at $\mathrm{T} \sim 600 \div 700 \mathrm{~K}$ is based on the decrease in the free path of the charge carriers as a result of thermal vibrations of the crystal lattice, the situation at $T \geq 700 \mathrm{~K} \mathrm{~K}$ was explained by the emergence of new recombination centers specific to localized traps. Polycrystalline silicon heated by sunlight does not create a barrier effect of traps localized in the grain boundary regions from polycrystalline silicon obtained by other methods. This can expand the possibilities of creating highly efficient semiconductor devices, solar cells, thermoelectric materials for micro- and nanoelectronics, photovoltaics.

\section{Introduction}

At present, the electrophysical and photoelectric properties of polycrystalline silicon semiconductors in the field when creating relatively inexpensive and resistant to external influences semiconductor devices, solar cells, thermoelectric materials can be considered sufficiently well studied from both experimental and theoretical points of view (see, for example, [1-11], as well as the links given there). It has been reliably established that the physical properties of polycrystalline silicon, which appear under certain conditions, depend on the area of the grain boundary of its volume. For example, localized traps or segregated impurity atoms in the grain boundary regions create a barrier effect that leads to shunting of the pn junction [1-10], and this simultaneously leads to a deterioration in the efficiency of semiconductor devices or solar cells. In this regard, one of the important tasks is the study of technologies for the development of polycrystalline semiconductor materials and the study of physical processes associated with grain boundary regions. There are a number of methods for obtaining polycrystalline silicon, of which the method of obtaining by heating in a solar oven [11] is the most attractive for polycrystalline semiconductors. In work [11], we experimentally proved and patented that the method makes it possible to obtain thermoelectric material based on granular silicon. This work is devoted to solving this problem for polycrystalline silicon obtained by heating in a solar oven using powder technology.

\section{Research Method}

Silicon samples heated in sunlight were selected for the study [11]. For the study of microstructures of images using X-ray spectral analysis, the measurement of specific resistance $(\rho)$ is carried out using modernized four-axis and Van der Pau methods $[9,10]$. Measurements of $\rho$ were carried out in a semiautomatic mode, both with an increase in temperature from $300 \mathrm{~K}$ to $800 \mathrm{~K}$ and at the stage of its lowering, i.e. in the process of cooling the samples.

\section{Results And Discussion}


In fig. 1 is a photomicrograph of a silicon sample heated by a sunbeam. As can be seen from the figure, the silicon sample has a polycrystalline structure with a rough grain surface of $10-20 \mu \mathrm{m}$. In fig. 2 also shows the X-ray spectral characteristics of impurity atoms. Crystal granularity was found to consist of $\mathrm{Si}$ atoms, and its surface was composed of roughness. The results obtained can be explained as follows.

It is known that the grain size and the formation of roughness on its surface depend on the technology for producing polycrystalline silicon. In the process of obtaining polycrystalline silicon, waste and uncontrolled impurities belonging to metallurgical silicon or impregnated with the external environment can be retained (for example, $\mathrm{Si}{ }^{\circledR} 98 \div 99 \%$; $\mathrm{Fe}, \mathrm{Au}, \mathrm{B}, \mathrm{P}, \mathrm{Ca}, \mathrm{Cr}, \mathrm{Cu}, \mathrm{Mg}, \mathrm{Mn}, \mathrm{Ni}, \mathrm{Ti}, \mathrm{V} \circledast 1 \div 2 \%$ ) [1-8]. These impurities can lead to the formation of defects of granular size and in the regions of inter grain boundaries, from point defects to complex shapes. For example, during the synthesis of silanetrichloride, $0.01 \div 1 \mathrm{~mm}$ of metallurgical silicon powder enters the reactor. In this process, various waste impurities atoms that enter through oxygen, residual water, and the external environment negatively affect the synthesis process. In order to get rid of it, special cleaning methods are used. However, is the found in the composition of purified $\mathrm{SiHCl} 3$ and semi-crystalline silicon, the content of group III and $\mathrm{V}$ elements to be $0,3 \times 10^{-7}$ and $<1,5 \times 10^{-7}$, respectively, and the amounts of heavy metals are $<0,1 \times 10^{-7}$, carbon $<300 \times 10^{-7}$, oxygen is $<50 \times 10^{-7}$, it was also found that the number of elements of III and $\mathrm{V}$ groups in the composition of the rod obtained by the zonal insertion method is $0,1 \times 10^{-7}$ and $<0,3 \times 10^{-7}$, respectively $[1-4,8]$.

In [4-10] it was noted that the grain of polycrystalline silicon consists of atoms $\mathrm{Si}$, and the roughness it its surface consists of residual impurity atoms. It was found that their amount increased from nucleus to surface, while conversely, the amount of silicon atoms decreased. In addition, it is shown in $[4,5,9]$ that the samples obtained by powder technology are fundamentally different from polycrystalline silicon

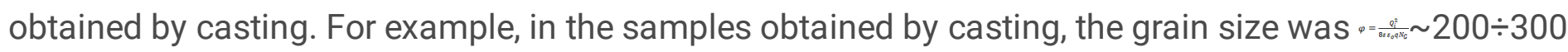
$\mu \mathrm{m}[4,9]$, and in the samples obtained by powder technology, it was $\sim 100 \mu \mathrm{m}[4,5]$, according to which the roughness structure on the granule surface was also different. This is explained on the basis that it depends on the chemical processes and thermodynamic conditions in the crystallization process. In the casting method, the raw material is carried out at or above the melting point of silicon. Crystallization due to thermodynamic changes is not the same in all parts, which leads to an uneven distribution of impurities, and this leads to the formation of various defects and between grain boundary regions in the crystal lattice. In powder technology, pressed samples are subjected to heat treatment close to the melting point of the material, for example, for silicon at temperatures of $\sim 1200 \div 1250^{\circ} \mathrm{C}[4,5]$. During heat treatment, the powders may melt but they will not liquefy. Therefore, they do not change the shape they take during the pressing process. The formation of an accumulation of impurities or the process of segregation is explained by the formation of roughness.

In our case, the samples were obtained by powder technology $[4,5,11]$. It should be noted that in contrast to traditional powder technology, in our case, no metallic substances, such as steel cylinders or balls, or similar substances are used in the powdering process [11]. The powder production process is carried out on ceramic materials with high hardness. This prevents contamination of silicon powder by the external environment as well as operations such as dust cleaning. According to this method, silicon particles are 
weighed in any desired medium, for example, oxygen, and using powder technology until they reach a powdery state with a size of up to 1 micrometer. The prepared silicon particles are washed with $70 \%$ ethyl alcohol, and a mixture of particles is formed. The prepared mixture (2) is poured into a container based on heat-resistant, for example, ceramic material (1) (Fig. 3). In this case, the mixture is pressed with little force and heated by the sun's rays. The heating of the mount with the help of sunlight is carried out in a solar furnace. The following parameters must be taken into account during the heating process: the thickness of the granular mixture on the base of the heat-resistant ceramic material should not exceed $2,5 \div 3 \mathrm{~mm}$; for silicon, the temperature should be $600 \div 1250^{\circ} \mathrm{C}$ [11].

It should be noted that when the thickness of the mixture is less than $2.5-3 \mathrm{~mm}$, instead of adhesion of particles, the degree of liquefaction increases. Conversely, when the thickness of the mixture is large, the temperature is not uniformly distributed throughout the volume. As a result, due to the temperature difference, aggregation in the surface areas occurs more slowly than in the lower part or in the volume, in contrast to the higher one. Attaching silicon particles by heating them with sunlight $(h g)$ is also a specific process. In this case, the mixture of silicon particles deposited on the substrate moves along the focal point of the solar oven device. That is, the mixture of silicon particles deposited on the substrate slowly moves from $600^{\circ} \mathrm{C}$ to $1200^{\circ} \mathrm{C}$ in a ray of sunlight, and this process takes 5-7 minutes. If the mixture of silicon particles is suddenly heated to a temperature of $1200^{\circ} \mathrm{C}$, the scattering of silicon particles is observed. The silicon particle mixture is therefore moved from the low temperature to the high temperature state along the focus of the solar furnace device (Fig. 3).

Thus, in the process of heating with sunlight, the silicon particles combine to form grains. This ensures that the crystal has a polycrystalline structure. It can be seen from the X-ray spectral characteristics of impurity atoms (Fig. 2) that grains $10-20 \mu \mathrm{m}$ in size consist of Si atoms. It should be noted that silicon particles $0.5-3 \mu \mathrm{m}$ in size were used to prepare the sample. In our opinion, small-sized silicon particles combine into relatively large-sized particles. In the work [4, 12], we proposed a structural model of silicon particles, obtained by us on the basis of powder technology. According to the model, the heating process is observed in silicon particles obtained by powder technology. The heating process does not occur uniformly across the particle crystal size. That is, there is a temperature difference from the particle core to the surface ( $T 3 \leq T 2 \leq T 1$, Fig. 4). In this case, the structure of the silicon particle can be divided into 3 parts according to the temperature difference (Fig. 4). The rough surface (1) has a relatively high temperature, the core of the particle (3) and consists of a separating region (2).

The surface area temperature is high, it consists of empty or broken bonds, and the energy of defect formation in the field also increases. In contrast to the nucleus, in the region close to the surface, the initial region of atomic decay of the crystal lattice appears (region 2). The atomic structure of this field is radically different from the atomic structure of fields 1 and 3 . In this case, the reactivity of the surface of a silicon particle increases from the center to the surface in accordance with its atomic structure $[4,12]$. According to the model, the reactivity of a small-sized particle nucleus is close to the reactivity of areas 1 and 2 of a large-sized particle. In this case, particles of small size accumulate around large particles. The result is areas of a certain size of granularity and between grain boundaries. 
It is known that the electrophysical properties of polycrystalline semiconductors are explained by charge transfer processes in the between grain boundary areas [1-10]. In fig. 5 illustrates the temperature dependence of the $r$. To compare the results obtained, we will use the results presented in $[4,5]$. The dependence of $r$ on the temperature change can be conditionally divided into $a-b, b-c$, and $c-d$.

In polycrystalline semiconductors, localized traps in the inter grain boundary area create a barrier effect. It also depends on the change in the height $r$ of the potential barrier $(j)$ during capture and charge emission in localized traps. According to the Setto model, the following expression can be written for $r[1,2]$ :

$$
\rho=\frac{k}{q\langle a\rangle A^{*} T} \exp \left(\frac{q \varphi}{k T}\right)
$$

here, $\mathrm{q}$ - electronic charge, $\mathrm{k}$ - Boltzmann constant, áañ - grain size, $A^{\star}$ - Richardson's efficiency constant, $\mathrm{T}$ - temperature, $\mathrm{j}$ - is the height of the potential barrier in the between grain boundary area.

It is known that $j$ depends on the amount of charges trapped in localized traps $\left(Q_{j}\right)$ :

$$
\varphi=\frac{Q_{i}^{2}}{8 \varepsilon \varepsilon_{o} q N_{G}}
$$

where is the $\mathrm{N}_{\mathrm{G}}$ - concentration of electrically active doped impurities, $\mathrm{e}$ and $\mathrm{e}_{\mathrm{o}}$ are the relative dielectric constant of the medium and the absolute dielectric constant of the vacuum, respectively.

(2) shows that the increase in charges $\left(Q_{i}\right)$ trapped in localized traps leads to an increase in $j$, which in turn leads to an increase in $r$ (case $a-b$ ', lines 2, 3, 4). However, the sample prepared by heating with sunlight $r$ (line 1) $[4,5]$ is radically different from the results given in the study (lines 2,3,4). That is, at $\mathrm{T} £ 600 \mathrm{~K} r$ decreases and then increases, at $\mathrm{T} \geq 700 \mathrm{~K}$ it stably changes (line 1 ).

It is known from semiconductor physics that the dependence of electrical conductivity on temperature change is expressed by the mobility and concentration of charge carriers. Under the influence of temperature, the concentration of charge carriers increases due to an increase in the concentration of ionized impurity atoms. This leads to an increase in permeability. At later stages of increasing the temperature, the impurity atoms are ionized, the concentration of charge carriers does not change, since the phonon energy is insufficient to ionize the main atoms of the semiconductor. It is also observed that the free running path of the charge carriers is reduced as a result of the thermal vibration of the crystal lattice. In this case, a decrease in the mobility of the charge carriers leads to a decrease in the conductivity. As the temperature increases further, the major atoms of the semiconductor ionize and a special conductivity is formed. 
The sample prepared by heating with sunlight (line 1, Fig. 5) confirms the observations given by the change in temperature $r$. In our opinion, at $\mathrm{f} £ 600 \mathrm{~K}$, the concentration of charge carriers increases due to an increase in the concentration of ionized impurity atoms, which, in turn, leads to a decrease in $r$ (case ab, line 1, Fig. 5). At T 600 $\div 700$, the decrease in the free path of the charge carriers as a result of thermal oscillations of the crystal lattice leads to an increase in $r$ (case b-c, line 1). At $T \geq 700 \mathrm{~K}$, a constant change in $r(\mathrm{c}-\mathrm{d}$ condition, line 1$)$ may be due to the appearance of new recombination centers specific to localized traps. For example, at T $600 \div 700 \mathrm{~K}$, oxygen atoms can diffuse to the surface, leading to the formation of various forms of silicon oxide $\left(\mathrm{SiO}_{x}, \mathrm{Si}_{y} \mathrm{O}_{x}\right)$ compounds on the surface of the silicon crystal [1-10, 13-15].

In this case, the Si-O bond energy is $4.6 \mathrm{eV}$, which is sufficient to break the Si-Si bond with an energy of $2.3 \mathrm{eV}$. In our opinion, thermal vibrations of the crystal lattice are enhanced, and as a result, oxygen diffuses to the surface with the formation of silicon oxide compounds [1-10, 13-15]. Although oxygen is electrically neutral in silicon, it has a significant effect on charge carriers during temperature changes. That is, as the ionization process increases, the $r$ at $T \geq 700 \mathrm{~K}$ changes steadily at the expense of oxygen. In other words, the results obtained for polycrystalline silicon heated by sunlight due to this mechanism are fundamentally different from the results obtained for polycrystalline silicon obtained by other methods.

\section{Conclusion}

Polycrystalline semiconductor materials form a barrier effect of localized traps or segregated impurity atoms in grain boundaries, which leads to shunting of the p-n junction [1-10]. It also has a negative effect on the efficiency of semiconductor devices or solar cells made on the basis of polycrystalline silicon. In our opinion, polycrystalline silicon heated by sunlight does not create a barrier effect of traps localized in the grain boundary regions from polycrystalline silicon obtained by other methods. This can expand the possibilities of creating highly efficient semiconductor devices, solar cells, thermoelectric materials for micro- and nanoelectronics, photovoltaics.

These results can be used to create highly efficient $\mathrm{p}-\mathrm{n}$ structures based on polycrystalline semiconductors, as well as to explain the electronic properties of the inter grain boundaries.

\section{Financing the work}

The work was done within India-Uzbekistan programme of co-operation in science \& technology "Development and implementation of micro- and nanoscale (granulated) semiconductor thermoelectric materials".

\section{Declarations}

* Ethics approval and consent to participate $-Y$ 
* Availability of data and materials $-Y$

* Competing interests - No

*Funding__ - No

* Authors' contributions $-Y$

* Acknowledgements

* Authors' information (optional).

Olimov Lutfiddin Omanovich*,

professor Andijan Machine Building institute

№56 Bobur Shox Ave, Andijan, Uzbekistan, 170100

ORCID 0000-0002-0135-5861

^email: O.Lut@mail.ru Tel: +998941089666

Anarboyev Iqboljon Ibroximovich,

PhD doctoral student Andijan Machine-Building Institute

№56 Bobur Shox Ave, Andijan, Uzbekistan, 170100

* Disclosure of potential conflicts of interest - No

* Research involving Human Participants and/or Animals - No

*Informed consent $-Y$

\section{References}

[1] Harbek G. 1989 (eds) Polycrystalline semiconductors. Physical properties and applications: (Moscow, Russia).

[2] L. Kazmerskii. 1983 (eds) Thin Polycrystalline and Amorphous Films. Physics and Applications. (Moscow, Russia).

[3] Vavilov, V.S., Kiselev, V.F., and Mukashev, B.N. 1990 (eds) Defects in Silicon and on Its Surface. (Moscow, Russia). 
[4] Olimov L.O. 2016 (eds) "The grain boundaries of polycrystalline silicon: microwaves, charge states and p-n-junction". Autoreferaty of doctoral dissertation. (Uzbekistan)

[5] Olimov L.O, Abdurakhmanov B.M, Teshaboev A. 2014 Influence of alkali metal atoms on transport of charge carriers in the between grain boundaries of polycrystalline silicon. J. Material Science, 114.

[6] Saidov, M.S., Abdurakhmanov, B.M., Olimov, L.O. 2007 Impurity thermovoltaic effect in the grain boundaries of a polycrystalline silicon solar cell. J. Applied Solar Energy, 4203.

[7] Olimov, L.O. 2012 Effect of alkali metals on the electronic properties of grain boundaries on a polycrystalline silicon surface. J. Semiconductors, 46898.

[8] Olimov, L.O. 2010 Adsorption of alkali metals and their effect on electronic properties of grain boundaries in bulk of polycrystalline silicon. J. Semiconductors, 44602.

[9] Olimov, L.O. 2010 Model of the grain boundary in p-n structures based on polycrystalline semiconductors. J. Applied Solar Energy, 46118.

[10] Abdurakhmanov, B.M., Olimov, L.O., Saidov, M.S. 2008 Electrophysical properties of solar polycrystalline silicon and its $n^{+}-p$ structures at elevated temperatures. J. Applied Solar Energy $\mathbf{4 4} 46$.

[11] Olimov L.O., Anarboev I.I., Mamirov A., Omonboev F.L., Omonboeva M.L. 2021 (eds) Patent UZ № FAP 01593 "Method of preparation of thermoelectric material".

[12] LO Olimov, ZM Sokhibova, BM Abdurakhmanov 2018 Some features of charge carrier transfer in granular semiconductors. I. Structure and mechanism of the phenomenon. International Journal of Advanced Research in Engineering and Applied Sciences. 71.

[13] Gnidenko A.A. and Zavodinsky V.G. 2008 Effect of Oxygen on Structure and Electronic Properties of Silicon Nanoclusters $\operatorname{Si}_{n}(n=5,6,10,18)$, J. Semiconductors 42800 .

[14] Bratus' V.Ya., Yukhimchuk V.A., Berezhinsky L.I., Valakh M.Ya., Vorona I.P., Indutnyi I.Z., Petrenko T.T., Shepeliavyæ P.E., and Yanchuk I.B. 2001 Structural Transformations and Silicon Nanocrystallite Formation in $\mathrm{SiO}_{x}$ Films, J. Semiconductors, 35821.

[15] Shigeki Imai and Masayuki Fujimoto 2006 Formation of (111) nanotwin lamellae hillocks in polycrystalline silicon thin films caused by deposition of silicon dioxide layer. J. Appl. Phys. Lett. 88 021912

\section{Figures}




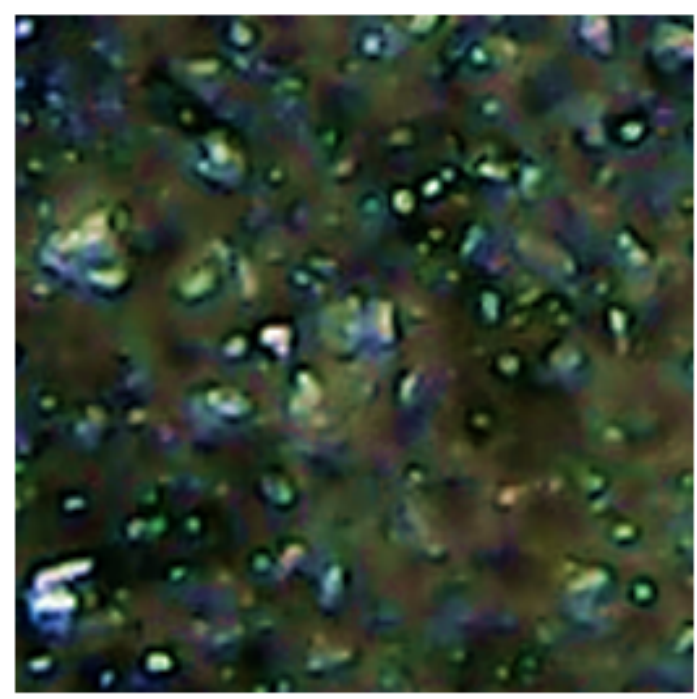

Figure 1

Microphotography of samples $(250 \times 250 \mu \mathrm{m})$.

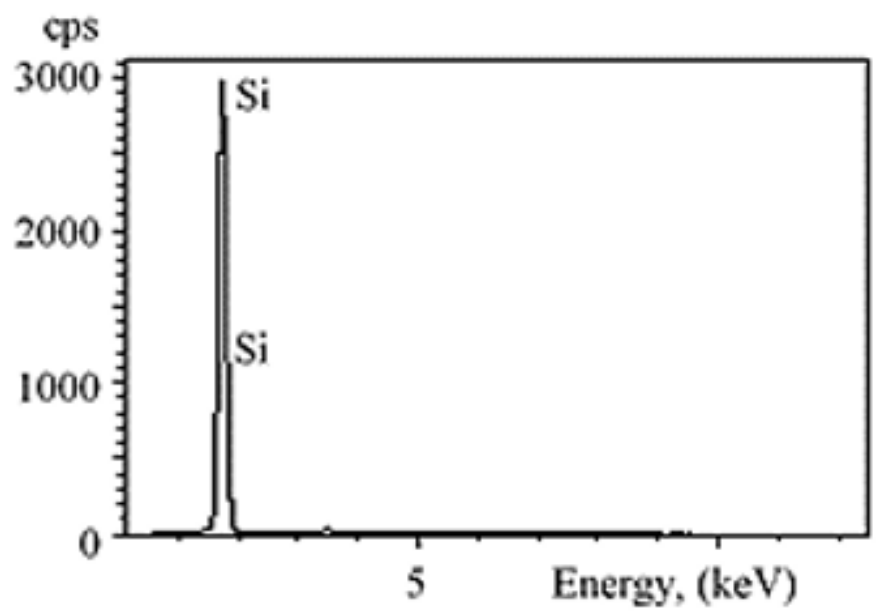

Figure 2

X-ray spectral characteristics of primary atoms of the image. 

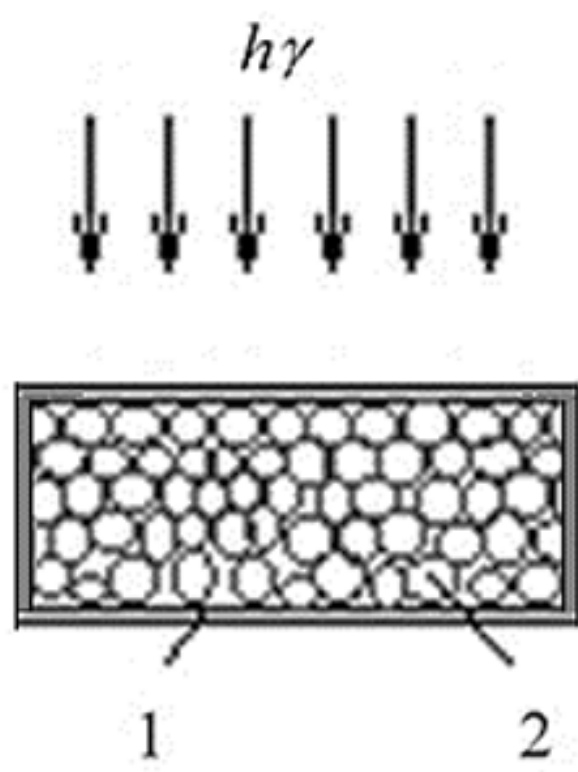

Figure 3

Scheme of bonding of silicon particles by heating with sunlight.

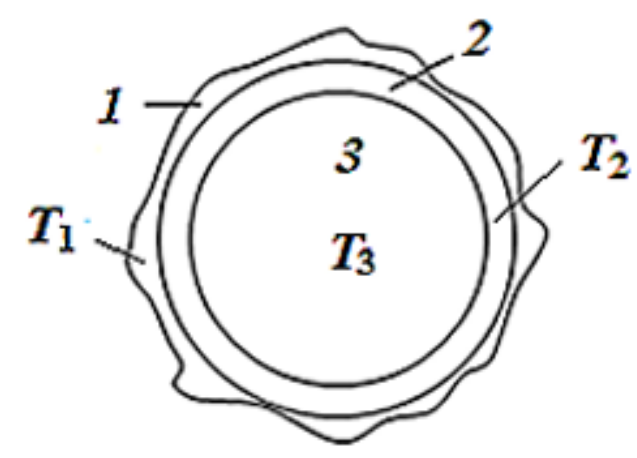

Figure 4

Simplified scheme of silicon particle; 1 - Rough surface area with uneven atomic structure, 3 - granule core, 2 - the area separating the core (1) from the field, $T 3 \leq T 2 \leq T 1$ is the temperature difference in the corresponding areas. 


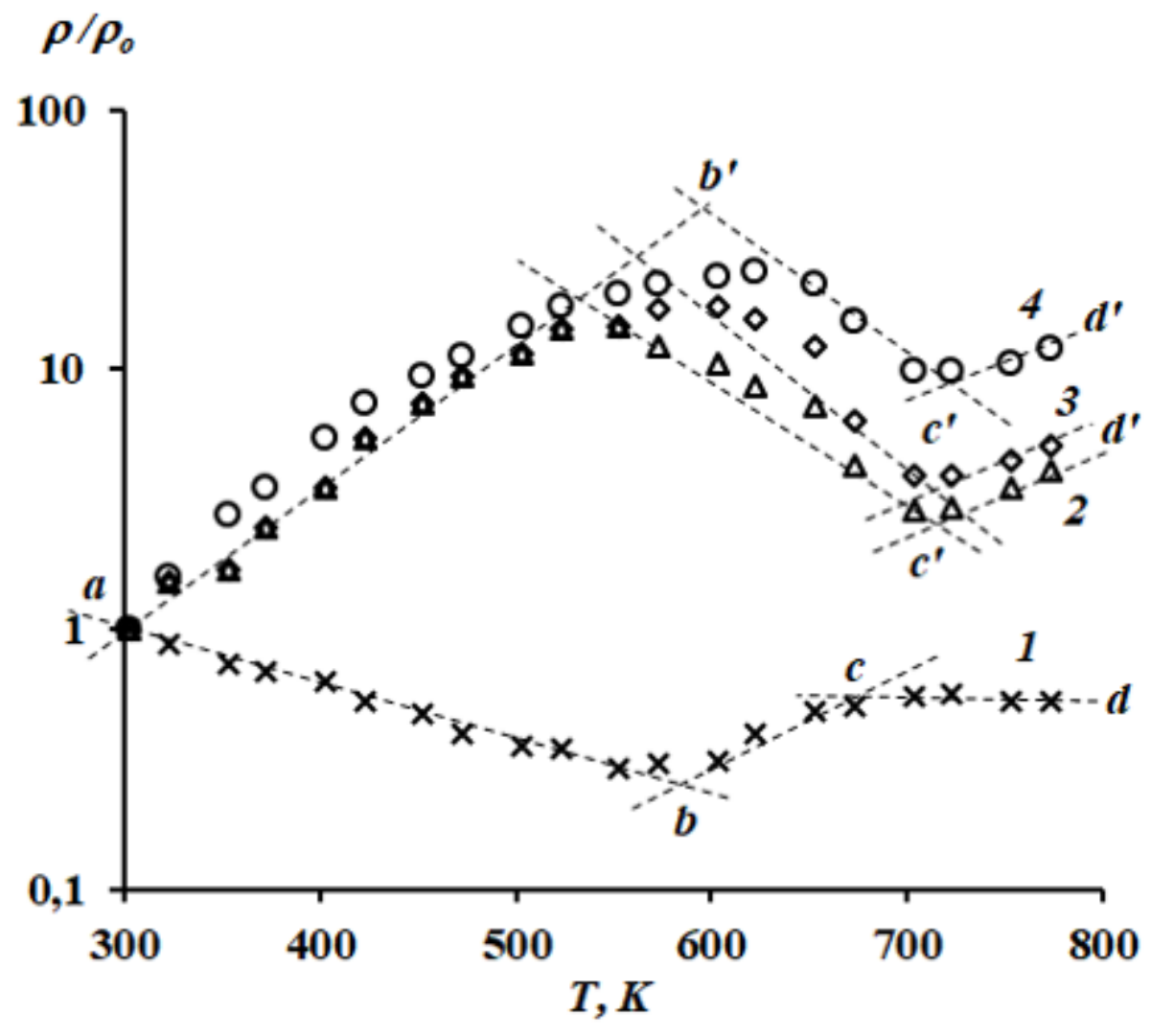

Figure 5

Temperature dependence of resistance. 